\title{
Two Market Models for Demand Response in Power Networks
}

\author{
Lijun Chen, Na Li, Steven H. Low and John C. Doyle \\ Engineering \& Applied Science Division, California Institute of Technology, USA
}

\begin{abstract}
In this paper, we consider two abstract market models for designing demand response to match power supply and shape power demand, respectively. We characterize the resulting equilibria in competitive as well as oligopolistic markets, and propose distributed demand response algorithms to achieve the equilibria. The models serve as a starting point to include the appliance-level details and constraints for designing practical demand response schemes for smart power grids.
\end{abstract}

\section{INTRODUCTION}

The usual practice in power networks is to match supply to demand. This is challenging because demand is highly timevarying. The utility company or generator needs to provision enough generation, transmission and distribution capacities for peak demand rather than the average. As a result, the power network has a low load factor and is underutilized most of the time, which is very costly. For example, the US national load factor is about $55 \%$, and $10 \%$ of generation and $25 \%$ of distribution facilities are used less than 400 hours per year, i.e., $5 \%$ of the time [1]. Shaping the demand to reduce the peak and smooth the variation can greatly improve power system efficiency and yield huge savings.

An alternative strategy improving efficiency and reducing cost is to match the supply. As the proportion of renewable sources such as solar and wind power steadily rises, power supply will also become highly time-varying. Matching the supply will become a more effective and common way to improve power system efficiency and reduce cost [2].

In this paper, we consider two abstract market models for designing demand response to match the supply and shape the demand, respectively. Specifically, in section III, we consider a situation where there is an inelastic supply deficit (or surplus) on electricity, and study a supply function bidding scheme for allocating load shedding (or load increasing) among different customers/users to match the supply. Each customer submits a parameterized "supply" function to the utility company, which will decide on a market-clearing price based on the bids of customers, and is committed to shed (or increase) its load according to its bid and the market-clearing price [3]. We show that in a competitive market where customers are price taking, the system achieves an efficient equilibrium that maximizes the social welfare. In an oligopolistic market where customers are price anticipating and strategic, the system achieves a unique Nash equilibrium that maximizes another additive, global objective function. Based on these optimization problem characterizations of the market equilibria, we propose iterative, distributed supply function bidding schemes for the demand response to achieve the equilibria.

In section IV, we consider a situation where power supply is elastic, but customers are subjected to realtime spot prices and will shift (and sometimes reduce) their demands accordingly. Each customer has a lower bound as well as an upper bound on the total electricity demand over a day. The customer will allocate its power usage for different times, so as to maximize its aggregate net utilities over a day. We show that when customers are price taking, the system achieves an efficient equilibrium that maximizes social welfare. When customers are price anticipating and strategic, the system achieves a unique Nash equilibrium that maximizes another global objective function. Again, based on the optimization problem characterizations of the market equilibria, we propose distributed algorithms for demand shaping to achieve the equilibria.

The aforementioned demand response schemes require timely two-way communications between the customers and the utility company and even direct communications between the customers. They also require certain computing capability of the customers. These communication and computing capabilities will become norm in future smart grids [1]. With the integration of state-of-the-art communication and computing technologies, future power systems will become more intelligent, more open, more autonomous, and with much greater user participation. Our demand response schemes are intended to apply in exactly such smart power grids.

\section{RELATED WORK}

There exists a huge amount of work on market models for various aspects of power networks. We briefly discuss those works that are directly relevant to this paper.

The supply function equilibrium model has been widely used in the analysis of markets in many industries. It assumes that each supplier submits a supply function to an auctioneer, who will set a uniform market clearing price. In a seminal paper [3], Klemperer and Meyer study the supply function equilibrium and give conditions for the existence and the uniqueness of the Nash equilibrium in supply functions under uncertain demand, and show that the equilibria are contained in a range of prices and allocations between the Cournot and the Bertrand equilibria.

The most notable application of the supply function equilibrium model is to the wholesale electricity markets, see, e.g., [4], [5], [6], [7], [8]. In this paper, instead of applying it to the electricity supply side, we apply the supply function equilibrium concept to pricing and allocation on the demand side to match the electricity supply, with a special form of parameterized supply functions that can enable a simple implementation of the iterative supply function bidding as an effective demand response scheme in power networks.

The model studied in section IV-B is a straightforward extension of the competitive equilibrium models for the power 
network, see, e.g., [9]. In addition to trading off the costs and utilities among different customers, we also consider trading off the costs and utilities over time, which incentivizes customers to shift their electricity usage.

\section{DEMAND RESPONSE: MATCHING THE SUPPLY}

In this section we consider a situation where there is a supply "deficit" or "surplus" on electricity. The deficit can be due to a decrease in power generation from, e.g., a wind or solar farm because of a change to worse weather condition, or an increase in power demand because of, e.g., a hot weather. The surplus can be due to an increase in power generation from, e.g., a wind or solar farm because of a change to better weather condition, or a decrease in power demand at, e.g., the late night time. We assume that it is very costly to increase the power supply in the case of a deficit or decrease the supply in the case of a surplus, i.e., the power supply is inelastic. If we have good estimation of electricity deficit or surplus (e.g., an hour ahead or a day ahead), we can match the supply by customers/users shedding or increasing their loads. In the following we focus on the case with a supply deficit and consider a bidding scheme for the demand response. The case with a supply surplus can be handled in the same way.

\section{A. System Model}

Consider a power network with a set $N$ of customers/users ${ }^{1}$ that are served by one utility company (or generator). Associated with each customer $i \in N$ is a load $q_{i}$ that it is willing to shed in a demand response system. We assume that the total load shed needs to meet a specific amount $d>0$ of electricity supply deficit, i.e.,

$$
\sum_{i} q_{i}=d
$$

Assume that customer $i$ incurs a cost (or disutility) $C_{i}\left(q_{i}\right)$ when it sheds a load of $q_{i}$. We assume that cost function $C_{i}(\cdot)$ is continuous, increasing, strictly convex, and with $C_{i}(0)=0$.

We consider a market mechanism for the load shedding allocation, based on supply function bidding [3]. For simplicity of implementation of the demand response scheme, we assume that each customer's "supply" function (for load shedding) is parameterized by a single parameter $b_{i} \geq 0, i \in N$, and takes the form of

$$
q_{i}\left(b_{i}, p\right)=b_{i} p, i \in N .
$$

The supply function $q_{i}\left(b_{i}, p\right)$ gives the amount of load customer $i$ is committed to shed when the price is $p$. The utility company will choose a price $p$ that clears the market, i.e.,

$$
\sum_{i} q_{i}\left(b_{i}, p\right)=\sum_{i} b_{i} p=d
$$

from which we get

$$
p(b)=\frac{d}{\sum_{i} b_{i}} .
$$

Here $b=\left(b_{1}, b_{2}, \cdots, b_{|N|}\right)$, the supply function profile.

\footnotetext{
${ }^{1}$ Here a customer/user can be a single residential or commercial customer, or represent a group of customers that acts as a single demand response entity.
}

Remark: Supply function as a strategic variable allows to adapt better to changing market conditions (such as uncertain demand) than does a simple commitment to a fixed price or quantity [3]. This is one reason we use supply function bidding, as we will further study demand response under uncertain power network conditions. The other motivation to use supply function is to respect practical informational constraints in the power network. A customer might not want to reveal its cost function because of incentive or security concerns, or the cost function may require a high description complexity, which means more communication/computation. A properlychosen parameterized supply function "controls" information revelation while demands less communication/computation.

\section{B. Optimal demand response}

In this subsection, we consider a competitive market where customers are price taking. Given price $p$, each customer $i$ maximizes its net revenue

$$
\max _{b_{i} \geq 0} \quad p q_{i}\left(b_{i}, p\right)-C_{i}\left(q_{i}\left(b_{i}, p\right)\right),
$$

where the first term is the customer $i$ 's revenue when it sheds a load of $q_{i}\left(b_{i}, p\right)$ at a price of $p$ and the second term is the cost incurred.

1) Competitive equilibrium: We now analyze the equilibrium of the demand response system. A competitive equilibrium for the demand response system is defined as a tuple $\left\{\left(b_{i}\right)_{i \in N}, p\right\}$, such that

$$
\begin{aligned}
\left(C_{i}^{\prime}\left(q_{i}\left(b_{i}, q\right)\right)-p\right)\left(\hat{b}_{i}-b_{i}\right) & \geq 0, \quad \forall \hat{b}_{i} \geq 0, \\
\sum_{i} q_{i}\left(b_{i}, q\right) & =d .
\end{aligned}
$$

Theorem 1: There exists a unique competitive equilibrium for the demand response system. Moreover, the equilibrium is efficient, i.e., it maximizes the social welfare:

$$
\begin{array}{ll}
\max _{q_{i} \geq 0} & \sum_{i}-C_{i}\left(q_{i}\right) \\
\text { s.t. } & \sum_{i} q_{i}=d .
\end{array}
$$

Proof: From equations (6)-(7), we have

$$
\begin{aligned}
\left(C_{i}^{\prime}\left(q_{i}\right)-p\right)\left(\hat{q}_{i}-q_{i}\right) & \geq 0, \forall \hat{q}_{i} \geq 0, \\
\sum_{i} q_{i} & =d .
\end{aligned}
$$

This is just the optimality condition of optimization problem (8)-(9) [10]. The uniqueness of the equilibrium follows from the fact that problem (8)-(9) and its dual are strictly convex.

2) Iterative supply function bidding: The social welfare problem (8)-(9) can be easily solved by the dual gradient algorithm [10]. This suggests an iterative, distributed supply function bidding scheme for demand response that achieves the market equilibrium.

At $k$-th iteration:

- Upon receiving price $p(k)$ announced by the utility company over a communication network, each customer $i$ updates its supply function, i.e., $b_{i}(k)$, according to

$$
b_{i}(k)=\left[\frac{\left(C_{i}^{\prime}\right)^{-1}(p(k))}{p(k)}\right]^{+},
$$


and submits it to the utility company over the communication network. Here ' + ' denotes the projection onto $\mathcal{R}^{+}$, the set of nonnegative real numbers.

- Upon gathering bids $b_{i}(k)$ from customers, the utility company updates the price according to

$$
p(k+1)=\left[p(k)-\gamma\left(\sum_{i} b_{i}(k) p(k)-d\right)\right]^{+},
$$

and announces the price $p(k+1)$ to the customers over the communication network. Here $\gamma>0$ is a constant stepsize.

When $\gamma$ is small enough, the above algorithm converges [10]. The scheme requires only light communication and computation, and will converge in short time with modern communication and computing technologies even for a very large network. The utility company and customers jointly run the market (i.e., the iterative bidding scheme) to find equilibrium price and allocation before the actual action of load shedding. The equilibrium price will be a market-clearing price, and the actual load shedding is "supplied" according to this price.

\section{Strategic demand response}

In this subsection, we consider an oligopoly market where customers know that price $p$ is set according to (4) and are strategic. Denote the supply function for all customers but $i$ by $b_{-i}=\left(b_{1}, b_{2}, \cdots, b_{i-1}, b_{i+1}, \cdots, b_{|N|}\right)$ and write $\left(b_{i}, b_{-i}\right)$ for the supply function profile $b$. Each customer $i$ chooses $b_{i}$ that maximizes

$$
\begin{aligned}
u_{i}\left(b_{i}, b_{-i}\right) & =p(b) q_{i}\left(p(b), b_{i}\right)-C_{i}\left(q_{i}\left(p(b), b_{i}\right)\right) \\
& =\frac{d^{2} b_{i}}{\left(\sum_{j} b_{j}\right)^{2}}-C_{i}\left(\frac{d b_{i}}{\sum_{j} b_{j}}\right) .
\end{aligned}
$$

This defines a demand response game among customers.

1) Game-theoretic equilibrium: We now analyze the equilibrium of the demand response game. The solution concept we use is the Nash equilibrium [11]. A supply function profile $b^{*}$ is a Nash equilibrium if, for all customers $i \in N, u_{i}\left(b_{i}^{*}, b_{-i}^{*}\right) \geq$ $u_{i}\left(b_{i}, b_{-i}^{*}\right)$ for all $b_{i} \geq 0$. We see that the Nash equilibrium is a set of strategies for which no player has an incentive to change unilaterally.

Lemma 2: If $b^{*}$ is a Nash equilibrium of the demand response game, then $\sum_{j \neq i} b_{j}^{*}>0$ for any $i \in N$.

Proof: We prove the result by contradiction. Suppose that it does not hold, and without loss of generality, assume that $\sum_{j \neq i} b_{j}^{*}=0$ for a customer $i$. Then, the payoff for the customer $i$ is $u_{i}\left(b_{i}^{*}, b_{-i}^{*}\right)=0$ if $b_{i}^{*}=0$, and $u_{i}\left(b_{i}^{*}, b_{-i}^{*}\right)=$ $d^{2} / b_{i}^{*}-C_{i}(d)$ if $b_{i}^{*}>0$. We see that when $b_{i}^{*}=0$, the customer $i$ has an incentive to increase it, and when $b_{i}^{*}>0$ the customer has an incentive to decrease it. Hence, there is no Nash equilibrium with $\sum_{j \neq i} b_{j}^{*}=0$.

The above Lemma also implies that at the Nash equilibrium at least two customers have $b_{i}^{*}>0$.

Let $B_{-i}=\sum_{j \neq i} b_{j}$. We have

$$
\begin{aligned}
\frac{\partial u_{i}\left(b_{i}, b_{-i}\right)}{\partial b_{i}} & =\frac{d^{2}\left(B_{-i}-b_{i}\right)}{\left(B_{-i}+b_{i}\right)^{3}}-\frac{d B_{-i}}{\left(B_{-i}+b_{i}\right)^{2}} C_{i}^{\prime}\left(\frac{d b_{i}}{B_{-i}+b_{i}}\right) \\
& =\frac{d^{2}}{\left(B_{-i}+b_{i}\right)^{2}}\left[\frac{B_{-i}-b_{i}}{B_{-i}+b_{i}}-\frac{B_{-i}}{d} C_{i}^{\prime}\left(\frac{d b_{i}}{B_{-i}+b_{i}}\right)\right]
\end{aligned}
$$

The first term in the square bracket is strictly decreasing in $b_{i}$ and the second term is strictly increasing in $b_{i}$. So, if $\frac{B_{-i}}{d} C_{i}^{\prime}(0) \geq 1, \frac{\partial}{\partial b_{i}} u_{i}\left(b_{i}, b_{-i}\right) \leq 0$ for all $b_{i}$, and $b_{i}=0$ maximizes the customer $i$ payoff $u_{i}\left(b_{i}, b_{-i}\right)$ for the given $b_{-i}$. If $\frac{B_{-i}}{d} C_{i}^{\prime}(0)<1, \frac{\partial}{\partial b_{i}} u_{i}\left(b_{i}, b_{-i}\right)=0$ only at one point $b_{i}>0$. Furthermore, note that $\frac{\partial}{\partial b_{i}} u_{i}\left(0, b_{-i}\right)>0$ and $\frac{\partial}{\partial b_{i}} u_{i}\left(B_{-i}, b_{-i}\right)<0$. So, this point $b_{i}$ maximizes the customer $i$ payoff $u_{i}\left(b_{i}, b_{-i}\right)$ for the given $b_{-i}$. Thus, at the Nash equilibrium for the demand response game, $b^{*}$ satisfies

$$
b_{i}^{*}=0,
$$

if $\frac{B_{-i}^{*}}{d} C_{i}^{\prime}(0) \geq 1$; and otherwise,

$$
\frac{B_{-i}^{*}-b_{i}^{*}}{B_{-i}^{*}+b_{i}^{*}}-\frac{B_{-i}^{*}}{d} C_{i}^{\prime}\left(\frac{d b_{i}^{*}}{B_{-i}^{*}+b_{i}^{*}}\right)=0 .
$$

Lemma 3: If $b^{*}$ is a Nash equilibrium of the demand response game, then $b_{i}^{*}<B_{-i}^{*}=\sum_{j \neq i} b_{j}^{*}$ for any $i \in N$, i.e., each customer will shed a load of less than $d / 2$ at the equilibrium.

Proof: The result holds when $b_{i}^{*}=0$. Note that the second term on the left hand side of equation (15) is positive. So the first term must be positive as well, which requires $B_{-i}^{*}>b_{i}^{*}$.

The following result follows directly from Lemma 3.

Corollary 4: No Nash equilibrium exists when $|N|=2$.

Theorem 5: Assume $|N|>2$. The demand response game has a unique Nash equilibrium. Moreover, the equlibrium solves the following convex optimization problem:

$$
\begin{aligned}
\max _{0 \leq q_{i}<d / 2} & \sum_{i}-D_{i}\left(q_{i}\right) \\
\text { s.t. } & \sum_{i} q_{i}=d,
\end{aligned}
$$

with

$$
\begin{gathered}
D_{i}\left(q_{i}\right)=\left(1+\frac{q_{i}}{d-2 q_{i}}\right) C_{i}\left(q_{i}\right)-\int_{0}^{q_{i}} \frac{d}{\left(d-2 x_{i}\right)^{2}} C_{i}\left(x_{i}\right) d x_{i} . \\
\text { Proof: } \\
\text { First, note that } \\
D_{i}^{\prime}\left(q_{i}\right)=\left(1+\frac{q_{i}}{d-2 q_{i}}\right) C_{i}^{\prime}\left(q_{i}\right),
\end{gathered}
$$

which is a positive, strictly increasing function in $b_{i} \in[0, d / 2)$. So, $D_{i}\left(q_{i}\right)$ is a strictly convex function in $[0, d / 2)$. Thus, the optimization problem (16)-(17) is a strictly convex problem and has a unique solution. Based on the optimality condition [10] and after a bit mathematical manipulation, the unique solution $q^{*}$ is determined by

$$
\begin{aligned}
\left(p^{*}-\left(1+\frac{q_{i}^{*}}{d-2 q_{i}^{*}}\right) C_{i}^{\prime}\left(q_{i}^{*}\right)\right)\left(q_{i}-q_{i}^{*}\right) & \leq 0, \forall q_{i}, \\
\sum_{i} q_{i}^{*} & =d, \\
p^{*} & >0 .
\end{aligned}
$$

Second, note that the Nash equilibrium condition (14)-(15) can be written compactly as

$$
\left(\frac{d}{B_{-i}^{*}+b_{i}^{*}}-\frac{B_{-i}^{*}}{B_{-i}^{*}-b_{i}^{*}} C_{i}^{\prime}\left(\frac{d b_{i}^{*}}{B_{-i}^{*}+b_{i}^{*}}\right)\right)\left(b_{i}-b_{i}^{*}\right) \leq 0, \forall b_{i} .
$$


Recall that the (Nash) equilibrium price $p^{*}=d / \sum_{i} b_{i}^{*}$ and (Nash) equilibrium allocation $q_{i}^{*}=b_{i}^{*} p^{*}$. We can write equation (23) as

$$
\left(p^{*}-\left(1+\frac{q_{i}^{*}}{d-2 q_{i}^{*}}\right) C_{i}^{\prime}\left(q_{i}^{*}\right)\right)\left(b_{i} p^{*}-q_{i}^{*}\right) \leq 0 .
$$

Note that at the Nash equilibrium, $p^{*}>0$ since $\sum_{i} b_{i}^{*}>0$ by Lemma 2 , and $b_{i}$ is arbitrary. So, the above equation is equivalent to equation (20). Thus, the Nash equilibrium of the demand response game satisfies the optimality condition (20)-(22), and solves the optimization problem (16)-(17). The existence and uniqueness of the Nash equilibrium follows from the fact that problem (16)-(17) admits a unique optimum.

Suppose that there are $n$ customers with $q_{i}^{*}>0$ at the Nash equilibrium. By Lemma 3, $n \geq 3$. There exists at least one customer $j$ such that $D_{j}^{\prime}\left(q_{j}^{*}\right)=p^{*}$ and $q_{j}^{*} \leq d / n$. Thus, $p^{*} \leq\left(1+\frac{1}{n-2}\right) C_{j}^{\prime}(d / n) \leq M$, where $M=\max _{i} D_{i}^{\prime}(d / n) \leq$ $\max _{i} D_{i}^{\prime}(d / 3)$. Let $h=\max _{i}\left(D_{i}^{\prime}\right)^{-1}(M)$, we have $q_{i}^{*} \leq h$ for all $i \in N$. Quantities $h-d /|N|$ and $h-d / n$ can be seen as measures of the heterogeneity in the system. For a homogeneous system where customers have the same disutility function, both measures equal zero. We can show that the Nash equilibrium price $p^{*} \leq(1+h /(d-2 h)) \bar{p}$, where $\bar{p}$ is the price at competitive equilibrium discussed in last subsection.

Remark: Theorem 5 can be seen as reverse-engineering from the game-theoretic equilibrium into a global optimization problem.

2) Iterative supply function bidding: By Theorem 5, we can solve the Nash equilibrium of the demand response game by solving convex optimization problem (16)-(17). This suggests the following iterative supply function bidding scheme to achieve the market equilibrium.

At $k$-th iteration:

- Upon receiving price $p(k)$ announced by the utility company over the communication network, each customer $i$ updates its supply function, i.e., $b_{i}(k)$, according to

$$
b_{i}(k)=\left[\frac{\left(D_{i}^{\prime}\right)^{-1}(p(k))}{p(k)}\right]^{+},
$$

and submits it to the utility company over the communication network.

- Upon gathering bids $b_{i}(k)$ from customers, the utility company updates the price according to

$$
p(k+1)=\left[p(k)-\gamma\left(\sum_{i} b_{i}(k) p(k)-d\right)\right]^{+},
$$

and announces price $p(k+1)$ to customers over the communication network.

Note that the distributed convergence to the Nash equilibrium is a difficult problem in general, because of informational constraints in the system. Here we involve the utility company in mediating strategic interaction among customers, see equation (26), in order to achieve the equilibrium in a distributed manner. The strategic action of the customer is also partially encapsulated in equation (25).

\section{DEMAND RESPONSE: SHAPING THE DEMAND}

In this section, we consider demand shaping by subjecting customers to realtime spot prices and incentivizing them to shift or even reduce their loads. In the following we study a utility optimization model, based on which propose distributed algorithms for demand shaping.

\section{A. System Model}

Consider a power network with a set $N$ of customers/users that are served by one utility company (or generator). Associated with each customer $i \in N$ is its power load $q_{i}(t)$ at time $t^{2}$ We assume that each customer $i$ has a minimum total power requirement in a day ${ }^{3}$

$$
\sum_{t=1}^{T} q_{i}(t) \geq \underline{Q}_{i}, \quad i \in N,
$$

corresponding to, e.g., basic daily routines; and a maximum total power requirement in a day

$$
\sum_{t=1}^{T} q_{i}(t) \leq \bar{Q}_{i}, \quad i \in N
$$

corresponding to, e.g., the total energy usage for a comfortable life style.

Assume that each customer $i$ attains a utility $U_{i}\left(q_{i}, t\right)$ when its power draw is $q_{i}$ at time $t$. The time-dependent utility models a general situation where the customer may have different power requirements at different times. We assume that $U_{i}\left(q_{i}, t\right)$ as a function of $q_{i}$ is continuously differentiable, strictly concave, increasing, with the curvatures bounded away from zero.

On the supply side, we assume that the utility company has a time-dependent cost of $C(Q, t)$ when it supplies power $Q$ at time $t$. The time-dependent cost function models a situation where energy generation cost might be different at different times. For example, when renewable energy such as solar is presented, the cost may depend on weather conditions, and a sunny hour may reduce the demand on power from a traditional power plant and result in a lower cost. The modeling of the cost function is an active research issue. Here we assume that the cost function $C(Q, t)$ as a function of $Q$ is strictly convex, with a positive, increasing marginal cost.

We assume that the objective of the utility company is to maximize its net revenue. Given price $p(t)$, it plans on its supply so as to solve the following maximization problem: ${ }^{4}$

$$
\max _{Q(t) \geq 0} \sum_{t \in T} Q(t) p(t)-C\left(Q_{i}(t), t\right),
$$

whose solution takes a simple form

$$
C^{\prime}(Q(t), t)=p(t), \quad t \in T .
$$

Note that the supply must equal the demand in the power network. So, the price $(p(t))_{t \in T}$ should settle down at a point that clears the market

\footnotetext{
${ }^{2}$ Note that we redefine the notation. In this section $q_{i}$ denotes the load of customer $i$, while in section III $q_{i}$ denotes the amount of load that customer $i$ is willing to shed.

${ }^{3}$ Each day is divided into $T$ timeslots of equal duration, indexed by $t \in$ $T=\{1,2, \cdots,|T|\}$

${ }^{4}$ Our focus in this paper is on the demand side. We thus do not consider possible strategic behaviors of the utility company or generator.
} 


$$
\sum_{i \in N} q_{i}(t)=Q(t), \quad t \in T .
$$

\section{B. Optimal demand response}

In this subsection, we consider a competitive market where customers are price taking. Given realtime spot price $p(t)$, customer $i$ allocates its energy usage to maximize its aggregate net utility subject to constraints (27)-(28):

$$
\begin{aligned}
\max _{q_{i}(t) \geq 0} & \sum_{t \in T} U_{i}\left(q_{i}(t), t\right)-q_{i}(t) p(t) \\
\text { s.t. } & \sum_{t \in T} q_{i}(t) \geq \underline{Q}_{i}, \quad i \in N \\
& \sum_{t \in T} q_{i}(t) \leq \bar{Q}_{i}, \quad i \in N .
\end{aligned}
$$

The above model captures two of the essential elements of demand response: realtime pricing and demand shifting. Demand shifting is achieved through optimizing over a certain period of time.

1) Competitive equilibrium: By introducing Lagrange multiplier $\underline{\lambda}_{i} \geq 0$ and $\bar{\lambda}_{i} \geq 0$ for constraints (33) and (34) respectively, the optimal $q(t)$ of the problem (32)-(34) is determined by the following conditions

$$
\begin{array}{r}
U_{i}^{\prime}\left(q_{i}(t), t\right)=p(t)+\bar{\lambda}_{i}-\underline{\lambda}_{i}, i \in N, t \in T, \\
\underline{\lambda}_{i}\left(\underline{Q}_{i}-\sum_{t=1}^{T} q_{i}(t)\right)=0, i \in N, \\
\bar{\lambda}_{i}\left(\sum_{t=1}^{T} q_{i}(t)-\bar{Q}_{i}\right)=0, i \in N .
\end{array}
$$

When $\underline{\lambda}_{i}>0$, i.e, constraint (33) is tight, the customer would equivalently pay a higher price than it should, in order to meet the minimum demand on power. When $\bar{\lambda}_{i}>0$, i.e, constraint (34) is tight, the customer would equivalently pay a lower price than it should, which can happen when the utility company subsidizes the customer to encourage electricity consumption.

A competitive equilibrium for the demand response system is defined as a triple $\left\{\left(q_{i}(t)\right)_{i \in N, t \in T},(Q(t))_{t \in T},(p(t))_{t \in T}\right\}$ that satisfies (35)-(37) and (30)-(31).

Theorem 6: There exists a unique competitive equilibrium for the demand response system. Moreover, the equilibrium is efficient, i.e., it maximizes the social welfare:

$$
\begin{aligned}
\max _{q_{i}(t) \geq 0} & \sum_{t \in T}\left\{\sum_{i \in N} U_{i}\left(q_{i}(t), t\right)-C\left(\sum_{i \in N} q_{i}(t), t\right)\right\} \\
\text { s.t. } & \sum_{t=1}^{T} q_{i}(t) \geq \underline{Q}_{i}, \quad i \in N \\
& \sum_{t=1}^{T} q_{i}(t) \leq \bar{Q}_{i}, \quad i \in N .
\end{aligned}
$$

Proof: Plugging equations (30) and (31) into equations (35)-(37), we get

$$
\begin{array}{r}
U_{i}^{\prime}\left(q_{i}(t), t\right)-C^{\prime}\left(\sum_{i} q_{i}(t), t\right)=\bar{\lambda}_{i}-\underline{\lambda}_{i}, i \in N, t \in T, \\
\underline{\lambda}_{i}\left(\underline{Q}_{i}-\sum_{t=1}^{T} q_{i}(t)\right)=0, i \in N, \\
\bar{\lambda}_{i}\left(\sum_{t=1}^{T} q_{i}(t)-\bar{Q}_{i}\right)=0, i \in N,
\end{array}
$$

which is just the optimality conditions for the social welfare problem. The uniqueness of equilibrium comes from the fact that the social welfare problem and its dual are strictly convex.

2) Distributed algorithm: The social welfare problem (38)(40) suggests a distributed algorithm to compute the market equilibrium, based on the gradient algorithm [10].

At $k$-th iteration:

- The utility company collects demands $\left(q_{i}^{k}(t)\right)_{t \in T}$ from each customer $i$ over the communication network, calculates the total demand $\left(Q^{k}(t)\right)_{t \in T}$ and the associated marginal cost

$$
p^{k}(t)=C^{\prime}\left(Q^{k}(t), t\right), t \in T,
$$

and announces $\left(p^{k}(t)\right)_{t \in T}$ to customers over the communication network.

- Each customer $i$ updates its demand $q_{i}^{k}(t)$ after receiving the update on price $p^{k}(t)$, according to

$$
q_{i}^{k+1}(t)=\left[q_{i}^{k}(t)+\gamma\left(U_{i}^{\prime}\left(q_{i}^{k}(t), t\right)-p^{k}(t)\right)\right]^{s_{i}},
$$

where where $\gamma>0$ is a constant stepsize, and ' $s_{i}$ ' denotes projection onto the set $S_{i}$ specified by constraints (27)(28). The projection operation is easy to do, as constraints (27)-(28) are local to customers.

When $\gamma$ is small enough, the above algorithm converges [10]. The utility company and customers jointly run the market (i.e., the above distributed algorithm) to decide on power loads and supply for each time $t$.

\section{Strategic demand response}

In this subsection, we consider an oligopoly market where customers know marginal cost (or supply curve) of the utility company and are strategic. We can model demand response problem as a game among customers: Given other customer power loads $\left(q_{-i}(t)\right)_{t \in T}=\left\{\left(q_{j}(t)\right)_{t \in T}, j \in N /\{i\}\right\}$, each customer $i$ chooses $q_{i}(t)$ that maximizes

$$
\sum_{t \in T} u_{i}\left(q_{i}(t), q_{-i}(t)\right)=\sum_{t \in T} U_{i}\left(q_{i}(t), t\right)-q_{i}(t) C^{\prime}\left(\sum_{i \in N} q_{i}(t), t\right),
$$

subject to constraints (27)-(28).

1) Game-theoretic equilibrium: We now analyze the equilibrium of the demand response game. Note that the marginal cost $C^{\prime}(\cdot)$ is positive and increasing by assumption. Thus, customer $i$ 's payoff $\sum_{t \in T} u_{i}\left(q_{i}(t), q_{-i}(t)\right)$ is concave in $\left(q_{i}(t)\right)_{t \in T}$. So, at the Nash equilibrium, $\left(q_{i}^{*}(t)\right)_{t \in T}$ satisfies

$\sum_{t \in T} \frac{\partial u_{i}\left(q_{i}^{*}(t), q_{-i}^{*}(t)\right)}{\partial q_{i}^{*}(t)}\left(q_{i}(t)-q_{i}^{*}(t)\right) \leq 0,\left(q_{i}(t)\right)_{t \in T} \in S_{i}$.

Theorem 7: The demand response game has a unique Nash equilibrium. Moreover, it solves the convex problem:

$$
\begin{array}{ll}
\max _{q_{i}(t)} & \sum_{t \in T}\left\{\sum_{i \in N} U_{i}\left(q_{i}(t), t\right)-q_{i}(t) C^{\prime}\left(\sum_{i \in N} q_{i}(t), t\right)\right\} \\
\text { s.t. } & \sum_{t=1}^{T} q_{i}(t) \geq \underline{Q}_{i}, \quad i \in N \\
& \sum_{t=1}^{T} q_{i}(t) \leq \bar{Q}_{i}, \quad i \in N .
\end{array}
$$


Proof: It is straightforward to check that the objective (45) is strictly concave, and the Nash equilibrium condition (44) is the optimality condition (variational inequality) for the convex problem (45)-(47). The theorem follows.

2) Distributed algorithm: The above optimization problem characterization of the Nash equilibrium suggests a distributed algorithm to compute the equilibrium.

At $k$-th iteration:

- Customers exchange information on their demands $\left(q_{i}^{k}(t)\right)_{t \in T}$ over the communication network. Each customer $i$ then calculates the total demand $\left(Q^{k}(t)\right)_{t \in T}$ and updates its demand $q_{i}^{k}(t)$, according to

$$
\begin{aligned}
q_{i}^{k+1}(t)= & {\left[q_{i}^{k}(t)+\gamma\left(U_{i}^{\prime}\left(q_{i}^{k}(t), t\right)-C^{\prime}\left(Q^{k}(t), t\right)\right.\right.} \\
& \left.\left.-p^{k}(t) C^{\prime \prime}\left(Q^{k}(t), t\right)\right)\right]^{s_{i}}
\end{aligned}
$$

where $\gamma>0$ is a constant stepsize.

Customers need to communicate with each other to jointly run the above algorithm to decide on their power usage at each time $t$. Note that we could also involve the utility company in mediating strategic interaction among customers, as in subsection III-C.2.

\section{NumERICAL EXAMPLES}

In this section, we provide numerical examples to complement the analysis in previous sections. We consider a simple power network with 10 customers that join in the demand response system. Due to the page limit, we will only report results on iterative supply function bidding proposed in section III. We assume that each customer $i$ has a cost function $C_{i}\left(q_{i}\right)=a_{i} q_{i}+h_{i} q_{i}^{2}$ with $a_{i} \geq 0$ and $h_{i}>0$. The electricity supply deficit is normalized to be 100 , and the values for parameters $a_{i}$ and $h_{i}$ used to obtain numerical results are randomly drawn from $[1,2]$ and $[2,6]$, respectively.

Figure 1 shows the evolution of the price and 5 customers' supply functions with stepsize $\gamma=0.02$ for optimal supply function bidding and for strategic supply function bidding, respectively. We see that the price and supply functions approach the market equilibrium quickly.

In order to study the impact of different choices of the stepsize on the convergence of the algorithms, we have run simulations with different stepsizes. We found that the smaller the stepsize, the slower the convergence, and the larger the stepsize, the faster the convergence but the system may only approach to within a certain neighborhood of the equilibrium, which is a general characteristic of any gradient based method. In practice, the utility company can first choose large stepsizes to ensure fast convergence, and subsequently reduce the stepsizes once the price starts oscillating around some mean value.

\section{CONCLUSION}

We have studied two market models for demand response in power networks. We characterize the resulting equilibria in competitive as well as oligopolistic markets, and propose distributed demand response schemes and algorithms to match electricity supply and to shape electricity demand accordingly.

As further research steps, we are characterizing efficiency loss of the game-theoretic equilibria. We will also search
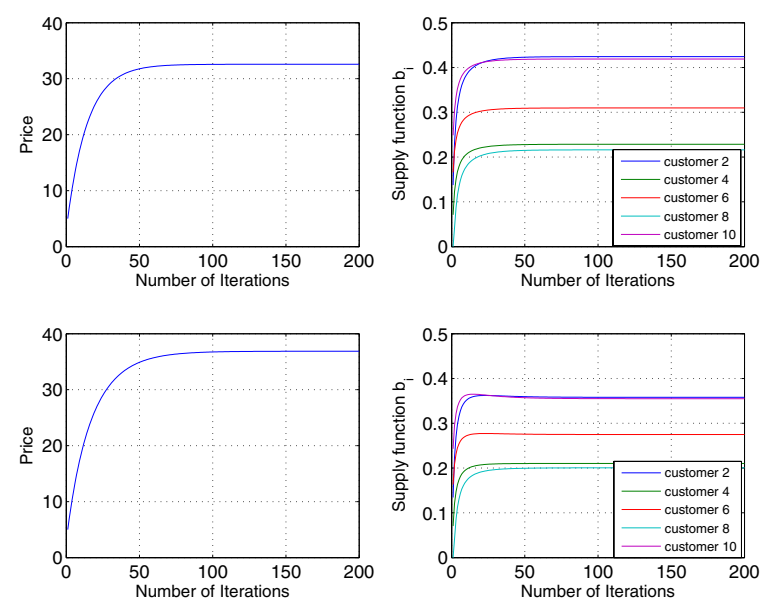

Fig. 1. Price and supply function evolution of optimal supply function bidding (upper panels) and strategic supply function bidding (lower panels) for demand response.

for other forms of parameterized supply functions that are more "expressive" while admit tractable analysis. As there are various uncertainties in power networks, e.g., it may be difficult to estimate or predict the power generation from the solar or wind farm precisely, we will study demand response under uncertain power network conditions.

This paper serves as a starting point for designing practical demand response schemes and algorithms for smart power grids. We will further bring in the detailed dynamics and realistic constraints of demand response appliances. We expect that these new constraints will not change the general structure of our models (in terms of, e.g, equilibrium characterization, and distributed decomposition structure, etc), but they will lead to higher communication overhead and computing complexity as we come to the scheduling of individual electronic appliances.

\section{REFERENCES}

[1] The Smart Grid: An Introduction. The US Department of Energy, 2008.

[2] B. Kirby and E. Hirst, Load as a resource in providing ancillary services, Technical report, Oak Ridge National Laboratory, 1999.

[3] P. D. Klemper and M. A. Meyer, Supply function equilibria in oligopoly under uncertainty, Econometrica, 57(6):1243-1277, 1989.

[4] R. Green and D. Newbery Competition in the british electricity spot market, Journal of Political Economy, 100(5):929-953, 1992.

[5] A. Rudkevich, M. Duckworth and R. Rosen, Modeling electricity pricing in a deregulated generation industry: The potential for oligopoly pricing in a Poolco, Energy Journal, 1998.

[6] R. Baldick and W. Hogan, Capacity constrained supply function equilibrium models of electricity markets: Stability, non-decreasing Constraints, and function space iterations, PWP-089 Working Paper, University of California Berkeley, 2002.

[7] R. Baldick, R. Grant and E. Kahn, Theory and application of linear supply function equilibrium in electricity markets, Journal of Regulatory Economics, 2004.

[8] R. Johari and J. Tsitsiklis, Parameterized supply function bidding: Equilibirium and welfare, Technical report, MIT, 2006.

[9] F. Wu, P. Varaiya, P. Spriller and S. Oren, Folk theorems on transmission access: Proofs and counterexamples, Journal of Regulatory Economics, 10(1):5-23, 1995 .

[10] D. P. Bertsekas and J. N. Tsitsiklis, Parallel and Distributed Computation, Prentice Hall, 1989.

[11] D. Fudenburg and J. Tirole, Game Theory, The MIT Press, 1991. 